\title{
Testing New Physics Explanations of the MiniBooNE Anomaly at Neutrino Scattering Experiments
}

\author{
Carlos A. Argüelles $\odot,{ }^{1, *}$ Matheus Hostert ${ }^{2, \dagger}$ and Yu-Dai Tsai ${ }^{3, \ddagger}$ \\ ${ }^{1}$ Department of Physics, Massachusetts Institute of Technology, Cambridge, Massachusetts 02139, USA \\ ${ }^{2}$ Institute for Particle Physics Phenomenology, Department of Physics, Durham University, \\ South Road, Durham DH1 3LE, United Kingdom \\ ${ }^{3}$ Fermilab, Fermi National Accelerator Laboratory, Batavia, Illinois 60510, USA
}

(Received 4 January 2019; revised manuscript received 17 October 2019; published 26 December 2019)

Heavy neutrinos with additional interactions have recently been proposed as an explanation to the MiniBooNE excess. These scenarios often rely on marginally boosted particles to explain the excess angular spectrum, thus predicting large rates at higher-energy neutrino-electron scattering experiments. We place new constraints on this class of models based on neutrino-electron scattering sideband measurements performed at MINER $\nu \mathrm{A}$ and CHARM-II. A simultaneous explanation of the angular and energy distributions of the MiniBooNE excess in terms of heavy neutrinos with light mediators is severely constrained by our analysis. In general, high-energy neutrino-electron scattering experiments provide strong constraints on explanations of the MiniBooNE observation involving light mediators.

DOI: 10.1103/PhysRevLett.123.261801

Introduction.-Nonzero neutrino masses have been established in the last twenty years by measurements of neutrino flavor conversion in natural and human-made sources, including long- and short-baseline experiments. The overwhelming majority of the data supports the threeneutrino framework. Within this framework, we have measured the mixing angles that parametrize the relationship between mass and flavor eigenstates to few-percentlevel precision [1]. The remaining unknowns are the absolute scale of neutrino masses and their origin, the $C P$-violating phase, and the mass ordering of the neutrinos. Nevertheless, anomalies in short-baseline accelerator and reactor experiments [2-5] challenge this framework and are yet to receive satisfactory explanations. Minimal extensions of the three-neutrino framework to explain the anomalies introduce the so-called sterile neutrino states, which do not participate in Standard Model (SM) interactions in order to agree with measurements of the Z-boson invisible decay width [6]. Unfortunately, these minimal scenarios are disfavored, as they fail to explain all data [7-10]. This has led the community to explore nonminimal scenarios. Along this direction, it is interesting to study wellmotivated neutrino-mass models that can also explain the short-baseline anomalies and are testable in the laboratory. In this work, we will investigate a class of

Published by the American Physical Society under the terms of the Creative Commons Attribution 4.0 International license. Further distribution of this work must maintain attribution to the author(s) and the published article's title, journal citation, and DOI. Funded by SCOAP . neutrino-mass-related models that have been proposed as an explanation of the anomalous observation of $\nu_{e}$-like events in MiniBooNE [5].

MiniBooNE is a mineral oil Cherenkov detector located in the Booster Neutrino Beam (BNB), at Fermilab [11,12]. Using data collected between 2002 and 2017, the experiment has observed an excess of $\nu_{e}$-like events that is currently in tension with the standard three-neutrino prediction and is beyond statistical doubt at the $4.7 \sigma$ level [5]. While it is possible that the excess is fully or partially due to systematic uncertainties or SM backgrounds (see, e.g., Refs. [13-15]), many beyond-the-Standard-Model (BSM) explanations have been put forth. These new physics (NP) scenarios typically require the existence of new particles, which can participate in short-baseline oscillations [16-37], change the neutrino propagation in matter [38-41], or be produced in the beam or in the detector and its surroundings [42-49]. These models either increase the conversion of muon- to electron-neutrinos or produce electron-neutrinolike signatures in the detector, where in the latter category one typically exploits the fact that the LSND and MiniBooNE are Cherenkov detectors that cannot distinguish between electrons and photons. Although many MiniBooNE explanations lack a connection to other open problems in particle physics, recent models [50-54] are motivated by neutrino-mass generation via hidden interactions in the heavy-neutrino sector. In particular, a common prediction of these models is the upscattering of a light neutrino into a heavy neutrino, usually with masses in the tens to hundreds of $\mathrm{MeV}$, which subsequently decays into a pair of electrons. To reproduce the MiniBooNE excess angular distribution, either the heavy 
neutrino must have moderate boost factors and the pair of electrons produced need to be collimated [51], or the heavy neutrino two-body decays must be forbidden [52].

In this Letter, we introduce new techniques to probe models that rely on the ambiguity between photons and electrons to explain the MiniBooNE observation, using the dark neutrino model from Refs. [50,51] as a benchmark scenario. Our analysis extends to all models with new marginally boosted particles produced in coherent-like neutrino interactions, as they predict large number of events at higher energies [42-49]. Thus, our analysis uses highenergy neutrino-electron scattering measurements [55-64]. This process is currently used to normalize the neutrino fluxes, due to its well-understood cross section, and has been a fertile ground for light NP searches [65-67]. Here, however, we expand the capability of these measurements to probe BSM-produced photon-like signatures, by developing a new analysis using previously neglected sideband data. Our technique is complementary to recent searches for coherent single-photon topologies [68]. Since the upscattering process has a threshold of tens to hundreds of $\mathrm{MeV}$, we focus on two high-energy neutrino experiments: MINER $\nu \mathrm{A}$ [58-61], a scintillator detector in the Neutrinos at the Main Injector (NuMI) beamline at Fermilab, and CHARM-II [62-64], a segmented calorimeter detector at CERN along the Super Proton Synchrotron (SPS) beam line. These experiments are complementary in the range of neutrino energies they cover and have different background composition. In all cases, a relevant sideband measurement exists, allowing us to take advantage of the excellent particle reconstruction capabilities of MINER $\nu \mathrm{A}$ and the precise measurements at CHARM-II to constrain NP.

Model.-We consider a minimal realization of dark neutrino models [50-54] that can explain MiniBooNE. This comprises one Dirac heavy neutrino, $\nu_{4}$, with its associated flavor state, $\nu_{D}$. (Models with the decay of Majorana particles will lead to greater tension with the angular distribution at MiniBooNE due to their isotropic nature $[69,70]$.) The dark neutrino $\nu_{D}$ is charged under a new local $U(1)^{\prime}$ gauge group, which is part of the particle content and gauge structure needed for mass generation. The dark sector is connected to the SM in two ways: kinetic mixing between the new gauge boson and hypercharge, and neutrino mass mixing. We start by specifying the kinetic part of the NP Lagrangian,

$$
\mathcal{L}_{\text {kin }} \supset \frac{1}{4} \hat{Z}_{\mu \nu}^{\prime} \hat{Z}^{\prime \mu \nu}+\frac{\sin \chi}{2} \hat{Z}_{\mu \nu}^{\prime} \hat{B}^{\mu \nu}+\frac{m_{\hat{Z}^{\prime}}^{2}}{2} \hat{Z}^{\prime \mu} \hat{Z}_{\mu}^{\prime},
$$

where $\hat{Z}^{\prime \mu}$ stands for the new gauge boson field, $\hat{Z}^{\prime \mu \nu}$ for its field strength tensor, and $\hat{B}^{\mu \nu}$ for the hypercharge field strength tensor. After usual field redefinitions [71], we arrive at the physical states of the theory. Working at leading order in $\chi$ and assuming $m_{Z^{\prime}}^{2} / m_{Z}^{2}$ to be small, we can specify the relevant interaction Lagrangian as

$$
\mathcal{L}_{\text {int }} \supset g_{D} \bar{\nu}_{D} \gamma_{\mu} \nu_{D} Z^{\prime \mu}+e \varepsilon Z^{\prime \mu} J_{\mu}^{\mathrm{EM}},
$$

where $J_{\mu}^{\mathrm{EM}}$ is the SM electromagnetic current, $g_{D}$ is the $U(1)^{\prime}$ gauge coupling assumed to be $\mathcal{O}(1)$, and $\varepsilon \equiv c_{\mathrm{w}} \chi$, with $c_{\mathrm{w}}$ being the cosine of the weak angle. Additional terms would be present at higher orders in $\chi$, and mass mixing with the SM $Z$ is also possible, though severely constrained. After electroweak symmetry breaking, $\nu_{D}$ is a superposition of neutrino mass states. The flavor and mass eigenstates are related via

$$
\nu_{\alpha}=\sum_{i=1}^{4} U_{\alpha i} \nu_{i}, \quad(\alpha=e, \mu, \tau, D)
$$

where $U$ is a $4 \times 4$ unitary matrix. It is expected that $\left|U_{\alpha 4}\right|$ is small for $\alpha=e, \mu, \tau$, but $\left|U_{D 4}\right|$ can be of $\mathcal{O}(1)[7,72]$.

MiniBooNE signature and region of interest.-The heavy neutrino is produced from an active flavor state upscattering on a nuclear target $A, \nu_{\alpha} A \rightarrow \nu_{4} A$. The upscattering cross section is proportional to $\alpha_{D} \alpha_{\mathrm{QED}} \varepsilon^{2}\left|U_{\alpha 4}\right|^{2}$, dominated by $\left|U_{\mu 4}\right|$, since all current accelerator neutrino beams are composed mainly of muon neutrinos. This production can happen off the whole nucleus in a coherent way or off individual nucleons. For $m_{Z^{\prime}} \lesssim 100 \mathrm{MeV}$, the production will be mainly coherent, but for heavier masses, such as the ones considered in Ref. [52], incoherent upscattering dominates. In Fig. 1, we show the NP cross section at the benchmark point of Ref. [51] and compare it with the quasielastic cross section. By superimposing the cross section on the neutrino fluxes of MINER $\nu \mathrm{A}$ and MiniBooNE, we make it explicit that the larger energies at MINER $\nu \mathrm{A}$ and CHARM-II are ideal to produce $\nu_{4}$. Once produced, $\nu_{4}$ predominantly decays into a neutrino and a

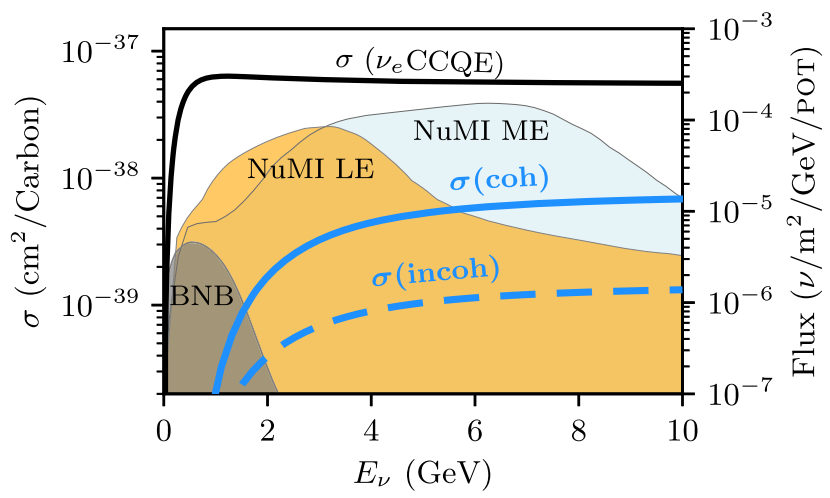

FIG. 1. Upscattering cross section compared to the quasielastic. The quasielastic cross section on carbon $\left(6 p^{+}\right)$is shown as a function of the neutrino energy (solid black line). The coherent (solid blue) and incoherent (dashed blue) scattering NP cross sections are also shown for the benchmark point of Ref. [51]. In the background, we show the BNB flux of $\nu_{\mu}$ at MiniBooNE (light gray), and the NuMI beam neutrino flux at MINER $\nu \mathrm{A}$ for the LE (light golden) and ME (light blue) runs in neutrino mode. 
dielectron pair, $\nu_{4} \rightarrow \nu_{\alpha} e^{+} e^{-}$, either via an on-shell [51] or off-shell [52] $Z^{\prime}$ depending on the choice of $m_{4}$ and $m_{Z^{\prime}}$. In this work, we restrict our discussion to the $m_{4}>m_{Z^{\prime}}$ case, where the upscattering is mainly coherent and is followed by a chain of prompt two-body decays $\nu_{4} \rightarrow \nu_{\alpha}\left(Z^{\prime} \rightarrow e^{+} e^{-}\right)$. The on-shell $Z^{\prime}$ is required to decay into an overlapping $e^{+} e^{-}$pair, setting a lower bound on its mass of a few $\mathrm{MeV}$. Experimentally, however, $m_{Z^{\prime}}>10 \mathrm{MeV}$ for $e \epsilon \sim 10^{-4}$ to avoid beam dump constraints [73]. Increasing $m_{Z^{\prime}}$ increases the ratio of incoherent to coherent events and makes the electron pair less overlapping. Even though we focus on overlapping $e^{+} e^{-}$pairs, we note that a significant fraction of events would appear as well-separated showers or as a pair of showers with large energy asymmetry, similarly to neutralcurrent (NC) $\pi^{0}$ events. The asymmetric events also contribute to the MiniBooNE excess and offer a different target for searches in $\nu-e$ scattering data.

A fit to the neutrino energy spectrum at MiniBooNE was performed in Ref. [51] and is reproduced in Fig. 3. We have performed our own fit to the MiniBooNE energy spectrum using the data release from Ref. [5], and our results agree with Ref. [51] when we simulate the signal at MiniBooNE and the analysis cuts in the same way. This fit leads to preferred values of $m_{4}$ close to $100 \mathrm{MeV}$ and $\left|U_{\mu 4}\right| \sim 10^{-4}$. Unfortunately, this energy-only fit neglects the distribution of the excess events as a function of their angle $\theta$ with respect to the beam. This is important, as the total observed excess contains only $\approx 50 \%$ of the events in the most forward bin $(0.8<\cos \theta<1.0)$, with a statistical uncorrelated uncertainty of $5 \%$ on this quantity.

As was recently pointed out in Ref. [74], few NP scenarios can reproduce the angular distribution of the MiniBooNE excess. Among these are models where new unstable particles are produced in inelastic collisions in the detector, such as the present case. Here, large $\theta$ can be achieved by tweaking the mass of the heavy neutrino; the signal becomes less forward as $\nu_{4}$ becomes heavier. To show this, we use our dedicated Monte Carlo (MC) simulation to assess the values of $m_{4}$ preferred by MiniBooNE data. (Since the released MiniBooNE data do not provide the correlation between angle and energy, and their associated systematics, an energy-angle fit is not possible.) For $m_{Z^{\prime}}=$ $30 \mathrm{MeV}$ and $m_{4}=100,200$, and $400 \mathrm{MeV}$, we find that $98 \%, 87 \%$, and $70 \%$ of the NP events would lie in the most forward bin, respectively. We find the predicted angular distribution to be more forward than Ref. [51] due to an improved MiniBooNE simulation; see the Supplemental Material for details [75]. This simulation discrepancy is understood and only strengthens our conclusions. Thus, the relevant region for the MiniBooNE angular distribution is $m_{4} \gtrsim 400 \mathrm{MeV}$ for $m_{Z^{\prime}}=30 \mathrm{MeV}$.

Our analysis.-Neutrino-electron scattering measurements predicate their cuts in the following core ideas: no hadronic activity near the interaction vertex, small opening angle from the beam, $E_{e} \theta^{2} \lesssim 2 m_{e}$, and the requirement that the measured energy deposition, $d E / d x$, be consistent with that of a single electron. For the NP events, when the coherent process dominates and the mass of the $Z^{\prime}$ is small, the first two conditions are often satisfied. However, the requirement of a single-electron-like energy deposition removes a significant fraction of the new-physics-induced events. This presents a challenge, as the NP events are mostly overlapping electron pairs and will potentially be removed by the $d E / d x$ cut. In order to circumvent this problem, we perform our analysis not at the final cut level, but at an intermediate one. This is done differently for CHARM-II and MINER $\nu$ A: the CHARM-II experiment provides data as a function of $E_{e} \theta^{2}$ without the $d E / d x$ cut, and MINER $\nu \mathrm{A}$ provides data as a function of the measured $d E / d x$ after analysis cuts on $E_{e} \theta^{2}$.

We have developed our own MC simulation for candidate electron pair events in MiniBooNE, MINER $\nu$ A, and CHARM-II; see the Supplemental Material for more details on detector resolutions, precise signal definition, and resulting distributions [75]. We only consider the coherent part of the cross section to avoid hadronic activity cuts, which is conservative. We also select only events with small energy asymmetries and small opening electron angles. When required, we assume the mean $d E / d x$ in the plastic scintillator to follow the same shape as the $\mathrm{NC} \pi^{0}$ prediction. Our prediction for new physics events for the BP point is shown in Fig. 2 on top of the MINER $\nu \mathrm{A}$ ME and CHARM-II data and MC prediction.

The CHARM-II analysis is mostly based on Fig. 1 of Ref. [64]. This sample is shown as a function of $E \theta^{2}$ and
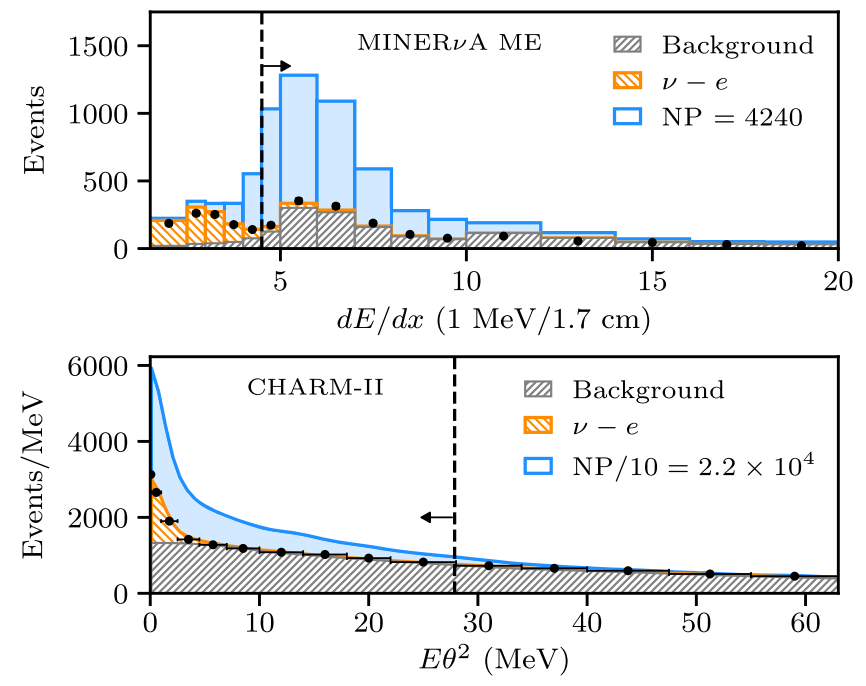

FIG. 2. New physics prediction at MINER $\nu \mathrm{A}$ ME and CHARM-II. Neutrino-electron scattering data in $d E / d x$ at MINER $\nu \mathrm{A}$ (top) and in $E \theta^{2}$ at CHARM-II (bottom). Error bars are too small to be seen. For both experiments, we show the $\nu-e$ signal and the total background prediction quoted (after tuning at $\mathrm{MINER} \nu \mathrm{A}$ ), as well as the NP prediction (divided by 10 at CHARM-II). The cuts in our analysis are shown as vertical lines. 
does not have any cuts on $d E / d x$. It contains all events with shower energies between 3 and $24 \mathrm{GeV}$, and our final cut on $E \theta^{2}$ is fixed at $28 \mathrm{MeV}$. For MINER $\nu \mathrm{A}$, the event selection is identical for the low-energy (LE) and medium-energy (ME) analyses $[59,60]$. The minimum shower energy required is $0.8 \mathrm{GeV}$ in order to remove the $\pi^{0}$ background and have reliable angular and energy reconstruction. Events are kept only when they meet the following angular separation criterion: $E_{e} \theta^{2}<3.2 \times 10^{-3} \mathrm{GeV} \mathrm{rad}^{2}$. A final cut is applied, ensuring $d E / d x<4.5 \mathrm{MeV} / 1.7 \mathrm{~cm}$. The MINER $\nu \mathrm{A}$ analyses use the data outside the previous $d E / d x$ cut to constrain backgrounds. This sideband is defined by all events with $E_{e} \theta^{2}>5 \times 10^{-3} \mathrm{GeV} \mathrm{rad}^{2}$ and $d E / d x<20 \mathrm{MeV} / 1.7 \mathrm{~cm}$. Using this sideband measurement, the collaboration tunes their backgrounds by $(0.76$, $0.64,1.0)$ for $\left(\nu_{e} \mathrm{CCQE}, \nu_{\mu} \mathrm{NC}, \nu_{\mu} \mathrm{CCQE}\right)$ processes in the LE mode. Our LE analysis uses the data shown in Fig. 3 of Ref. [60], where all the cuts are applied except for the final $d E / d x$ cut. In our final event selection, we require that the sum of the energy deposited be more than $4.5 \mathrm{MeV} / 1.7 \mathrm{~cm}$, compatible with an $e^{+} e^{-}$pair and yielding an efficiency of $90 \%$.

The recent MINER $\nu \mathrm{A}$ ME data contain an excess in the region of large $d E / d x$ [59], where the NP events would lie. However, this excess is attributed to NC $\pi^{0}$ events, and grows with the shower energy, undershooting the rate required to explain the MiniBooNE anomaly. With normalization factors as large as 1.7, the collaboration tunes primarily the NC $\pi^{0}$ prediction in an energy-dependent way. After tuning, the total NC $\pi^{0}$ sample corresponds to $20 \%$ of the total number of events before the $d E / d x$ cut.

To place our limits, we perform a rate-only analysis by means of a Pearson's $\chi^{2}$ as a test statistic; a detailed definition is given in the Supplemental Material [75]. We incorporate uncertainties in background size and flux normalization as nuisance parameters with Gaussian constraint terms. For the neutrino-electron scattering and BSM signal, we allow the normalization to scale proportionally to the same flux uncertainty parameter. The background term also scales with the flux-uncertainty parameter but has an additional nuisance parameter to account for its unknown size. We obtain our constraint as a function of heavy neutrino mass $m_{4}$, and mixing $\left|U_{\mu 4}\right|$ assuming a $\chi^{2}$ with 2 degrees of freedom [76].

In our nominal MINER $\nu \mathrm{A}$ LE (ME) analysis, we allow for $10 \%$ uncertainty on the flux [77], and 30\% (40\%) uncertainty on the background motivated by the amount of tuning performed on the original backgrounds. Note that the nominal background predictions in the MINER $\nu \mathrm{A}$ LE (ME) analysis overpredict (underpredict) the data before tuning, and that tuning parameters are measured at the $3 \%$ $(5 \%)$ level $[58,59]$. We also perform a background-ignorant analysis in which we assume $100 \%$ uncertainty for the background normalization, which changes our conclusions by only less than a factor of 2 . This emphasizes the robustness of our MINER $\nu \mathrm{A}$ bound, since the NP typically overshoots the low number of events in the sideband. For the benchmark point of Ref. [51], we predict a total signal of 232 (4240) events for MINER $\nu$ A LE (ME).

For CHARM-II, the NP signal lies mostly in a region with small $E \theta^{2}$. Thus, we constrain backgrounds using the data from $28<E \theta^{2}<60 \mathrm{MeV} \mathrm{rad}^{2}$. This sideband measurement constrains the normalization of the backgrounds in the signal region at the level of 3\%. The extrapolation of the shape of the background to the signal region introduces the largest uncertainty in our analysis. For this reason, we raise the uncertainty of the background normalization from $3 \%$ to a conservative $10 \%$ when setting the limits. Flux uncertainties are assumed to be $4.7 \%$ and $5.2 \%$ for the neutrino and antineutrino modes [78], respectively, and are applicable to the new-physics signal, $\nu-e$ scattering prediction, and backgrounds. Uncertainties in the $\nu-e$ scattering cross sections are expected to be subdominant and are neglected in the analysis [79]. For CHARM-II, the $\mathrm{NP}$ also yields too many events in the signal region, namely $\approx 2.2 \times 10^{5}$ events for the benchmark point of Ref. [51] in antineutrino mode. If we lower $\left|U_{\mu 4}\right|=10^{-4}$ and $m_{4}=100 \mathrm{MeV}$, CHARM-II would still have $\approx 3 \times 10^{3}$ new physics events.

Results and conclusions.-The resulting limits on dark neutrinos using neutrino-electron scattering experiments are shown in the $\left|U_{\mu 4}\right|$ vs $m_{4}$ plane at a $90 \%$ confidence level (C.L.) in Fig. 3. The MiniBooNE fit from Ref. [51] is shown, together with vertical lines indicating the percentage of events at MiniBooNE that populate the most forward angular bin. We have chosen the same values of $\varepsilon, \alpha_{D}$, and $m_{Z^{\prime}}$ as used in Ref. [51], and shown their benchmark point $\left(m_{4}=420 \mathrm{MeV}\right.$ and $\left.\left|U_{\mu 4}\right|^{2}=9 \times 10^{-7}\right)$ as a dotted circle. For these parameters, we can conclude that a good angular distribution at MiniBooNE is in large tension with neutrino-electron scattering data. We note that the MiniBooNE event rate scales identically to our signal rate in all the couplings, and the dependence on $m_{Z^{\prime}}$ is subleading due to the typical momentum transfer to the nucleus, provided $m_{Z^{\prime}} \lesssim 100 \mathrm{MeV}$. This implies that changing the values of these parameters does not modify the overall conclusions of our work. In addition, for this realization of the model, larger $m_{Z^{\prime}}$ implies larger values of $m_{4}$, increasing their impact on neutrino-electron scattering data. Our MINER $\nu \mathrm{A}$ and CHARM-II results are mutually reinforcing, given that they impose similar constraints for $m_{4} \lesssim 200 \mathrm{MeV}$. For larger masses, the kinematics of the signal becomes less forward, and the production thresholds start being important. This explains the upturns visible in our bounds, where we observe it first in MINER $\nu \mathrm{A}$ and later in CHARM-II as we increase $m_{4}$, since CHARM-II has higher beam energy.

We emphasize that our analysis is general, and can be adapted to other models. In fact, any MiniBooNE explanation with heavy new particles faces severe constraints from high-energy neutrino-electron scattering data if the 


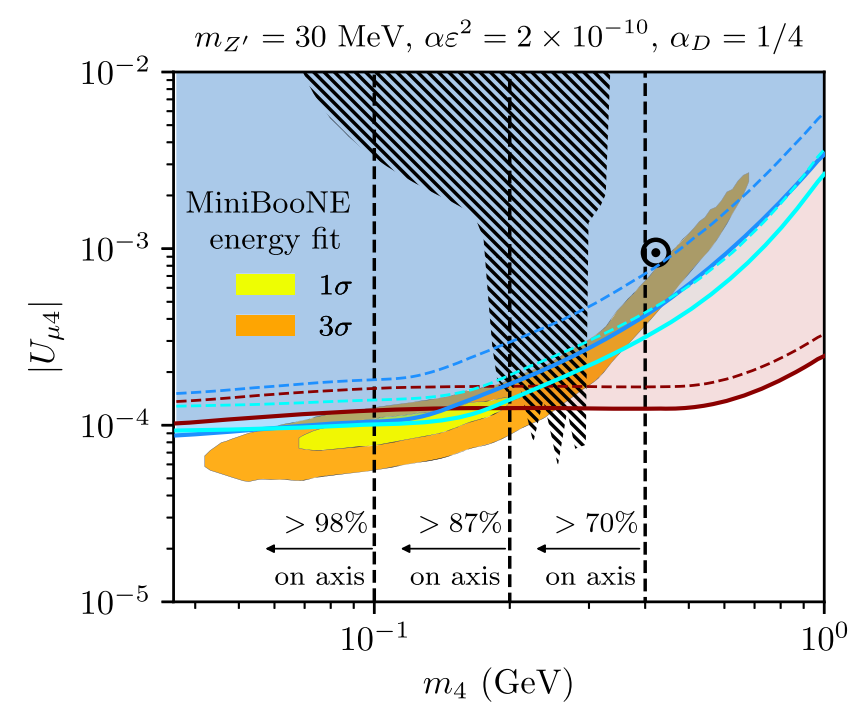

FIG. 3. New constraints on dark neutrinos as a MiniBooNE explanation. The fit to the MiniBooNE energy distribution from Ref. [51] is shown as the closed yellow (orange) region for a $1 \sigma$ $(3 \sigma)$ C.L., together with the benchmark point $(\odot)$. Our constraints are shown at a $90 \%$ C.L. for MINER $\nu$ A LE in blue (solid: $30 \%$ background normalization uncertainty; dashed: conservative $100 \%$ case), for MINER $\nu \mathrm{A}$ ME in cyan (solid: $40 \%$ background normalization uncertainty; dashed: conservative $100 \%$ case), and for CHARM-II in red (solid: 3\% background normalization from the sideband constraint; dashed: conservative $10 \%$ case). Vertical lines show the percentage of excess events at MiniBooNE that lie in the most forward angular bin. Exclusion from heavy neutrino searches is shown as a hatched background. Other relevant assumed parameters are shown above the plot; changing them does not alter our conclusion.

signal is free from hadronic activity. This is realized, for instance, in scenarios with heavy neutrinos with dipole interactions [42-49]. Our bounds can also be adapted to other scenarios with dark neutrinos and heavy mediators $[52,54]$. For those, however, we do not expect our bounds to constrain the region of parameter space where the MiniBooNE explanation is viable, since most of the signal at MiniBooNE contains hadronic activity which would be visible at MINER $\nu \mathrm{A}$ and CHARM-II.

In the near future, our new analysis strategy could be used in the upcoming MINER $\nu \mathrm{A}$ ME results on antineutrino-electron scattering. The NP cross section, being the same for neutrinos and antineutrinos, is thus more prominent on top of backgrounds. This class of analyses will also greatly benefit from improved calculations and measurements of coherent $\pi^{0}$ production and single-photon-emitting processes. This is particularly important given the excess seen in the MINER $\nu \mathrm{A}$ ME analysis. A new result can also be obtained by neutrino-electron scattering measurements at $\mathrm{NO} \nu \mathrm{A}$, which will sample a different kinematic regime as its off-axis beam peaks at lower energies and expects fewer $\mathrm{NC} \pi^{0}$ events per ton. Beyond neutrinoelectron scattering, the BSM signatures we consider could be lurking in current measurements of $\pi^{0}$ production, e.g., at MINOS [80] and MINER $\nu \mathrm{A}$ [81], and in analyses like the single-photon search performed by T2K [68]. (The $\nu_{e}$ $\mathrm{CCQE}$ measurement by MINER $\nu \mathrm{A}$ observes a significant excess of single photon-like showers attributed to diffractive $\pi^{0}$ events. These are abundant in similar realizations of this NP model [52].) Thus, if dark neutrinos are indeed present in current data, our technique will be crucial to confirm it.

To summarize, a variety of measurements are underway to further lay siege to this explanation of the MiniBooNE observation and, simultaneously, start probing testable neutrino mass generation models, as well as other similar NP signatures.

We thank Janet Conrad, Kareem Farrag, Alberto Gago, Gordan Krnjaic, Trung Le, Pedro Machado, Kevin Mcfarland, and Jorge Morfin for useful discussions, and Jean DeMerit for carefully proofreading our work. The authors would like to thank Fermilab for the hospitality at the initial stages of this project. Also, the authors would like to thank Fermilab Theory Group and the CERN Theory Neutrino Platform for organizing the conference "Physics Opportunities in the Near DUNE Detector Hall," which was essential to the completion of this work. C. A. A. would especially like to thank Fermilab Center for Neutrino Physics summer visitor program for funding his visit. M. H.'s work was supported by Conselho Nacional de Ciência e Tecnologia (CNPq). C. A. A. is supported by U.S. National Science Foundation (NSF) Grant No. PHY-1801996. This document was prepared by Y.-D. T. using the resources of the Fermi National Accelerator Laboratory (Fermilab), a U.S. Department of Energy, Office of Science, HEP User Facility. Fermilab is managed by Fermi Research Alliance, LLC (FRA), acting under Contract No. DE-AC02-07CH11359.

*caad@mit.edu

matheus.hostert@durham.ac.uk *ytsai@fnal.gov

[1] I. Esteban, M. C. Gonzalez-Garcia, A. Hernandez-Cabezudo, M. Maltoni, and T. Schwetz, Global analysis of threeflavour neutrino oscillations: Synergies and tensions in the determination of $\theta_{2} 3, \delta_{C} P$, and the mass ordering, J. High Energy Phys. 01 (2019) 106.

[2] C. Athanassopoulos et al. (LSND Collaboration), Evidence for $\bar{\nu}_{\mu} \rightarrow \bar{\nu}_{e}$ Oscillations from the LSND Experiment at LAMPF, Phys. Rev. Lett. 77, 3082 (1996).

[3] A. Aguilar-Arevalo et al. (LSND Collaboration), Evidence for neutrino oscillations from the observation of antineutrino(electron) appearance in a anti-neutrino(muon) beam, Phys. Rev. D 64, 112007 (2001).

[4] A. A. Aguilar-Arevalo et al. (MiniBooNE Collaboration), A Search for Electron Neutrino Appearance at the $\Delta m^{2} \sim$ $1 \mathrm{eV}^{2}$ Scale, Phys. Rev. Lett. 98, 231801 (2007). 
[5] A. A. Aguilar-Arevalo et al. (MiniBooNE Collaboration), Observation of a Significant Excess of Electron-Like Events in the MiniBooNE Short-Baseline Neutrino Experiment, Phys. Rev. Lett. 121, 221801 (2018).

[6] LEP Electroweak Working Group (ALEPH, CDF, D0, DELPHI, L3, OPAL, SLD, LEP Electroweak Working Group, Tevatron Electroweak Working Group, SLD Electroweak and Heavy Flavour Groups), Precision Electroweak Measurements and Constraints on the Standard Model, arXiv:1012.2367.

[7] G. H. Collin, C. A. Argüelles, J. M. Conrad, and M. H. Shaevitz, First Constraints on the Complete Neutrino Mixing Matrix with a Sterile Neutrino, Phys. Rev. Lett. 117, 221801 (2016).

[8] F. Capozzi, C. Giunti, M. Laveder, and A. Palazzo, Joint short- and long-baseline constraints on light sterile neutrinos, Phys. Rev. D 95, 033006 (2017).

[9] M. Dentler, Á. Hernández-Cabezudo, J. Kopp, P. A. N. Machado, M. Maltoni, I. Martinez-Soler, and T. Schwetz, Updated global analysis of neutrino oscillations in the presence of eV-scale sterile neutrinos, J. High Energy Phys. 08 (2018) 010.

[10] A. Diaz, C. A. Argüelles, G. H. Collin, J. M. Conrad, and M. H. Shaevitz, Where are we with light sterile neutrinos? arXiv:1906.00045.

[11] A. A. Aguilar-Arevalo et al. (MiniBooNE Collaboration), The neutrino flux prediction at MiniBooNE, Phys. Rev. D 79, 072002 (2009).

[12] A. A. Aguilar-Arevalo et al. (MiniBooNE Collaboration), The MiniBooNE Detector, Nucl. Instrum. Methods Phys. Res., Sect. A 599, 28 (2009).

[13] A. A. Aguilar-Arevalo et al. (MiniBooNE Collaboration), Unexplained Excess of Electron-Like Events from a 1-GeV Neutrino Beam, Phys. Rev. Lett. 102, 101802 (2009).

[14] A. A. Aguilar-Arevalo et al. (MiniBooNE Collaboration), A Combined $\nu_{\mu} \rightarrow \nu_{e}$ and $\bar{\nu}_{\mu} \rightarrow \bar{\nu}_{e}$ Oscillation Analysis of the MiniBooNE Excesses, Phys. Rev. Lett. 110, 161801 (2013).

[15] R. J. Hill, On the single photon background to $\nu_{e}$ appearance at MiniBooNE, Phys. Rev. D 84, 017501 (2011).

[16] H. Murayama and T. Yanagida, LSND, SN1987A, and CPT violation, Phys. Lett. B 520, 263 (2001).

[17] A. Strumia, Interpreting the LSND anomaly: Sterile neutrinos or CPT violation or...?, Phys. Lett. B 539, 91 (2002).

[18] G. Barenboim, L. Borissov, and J.D. Lykken, CPT violating neutrinos in the light of KamLAND, arXiv: hep-ph/0212116.

[19] M. C. Gonzalez-Garcia, M. Maltoni, and T. Schwetz, Status of the CPT violating interpretations of the LSND signal, Phys. Rev. D 68, 053007 (2003).

[20] V. Barger, D. Marfatia, and K. Whisnant, LSND anomaly from $C P T$ violation in four neutrino models, Phys. Lett. B 576, 303 (2003).

[21] M. Sorel, J. M. Conrad, and M. H. Shaevitz, A combined analysis of short baseline neutrino experiments in the $(3+1)$ and $(3+2)$ sterile neutrino oscillation hypotheses, Phys. Rev. D 70, 073004 (2004).

[22] G. Barenboim and N.E. Mavromatos, CPT violating decoherence and LSND: A possible window to Planck scale physics, J. High Energy Phys. 01 (2005) 034.
[23] K. M. Zurek, New matter effects in neutrino oscillation experiments, J. High Energy Phys. 10 (2004) 058.

[24] D. B. Kaplan, A.E. Nelson, and N. Weiner, Neutrino Oscillations as a Probe of Dark Energy, Phys. Rev. Lett. 93, 091801 (2004).

[25] H. Pas, S. Pakvasa, and T. J. Weiler, Sterile-active neutrino oscillations and shortcuts in the extra dimension, Phys. Rev. D 72, 095017 (2005).

[26] A. de Gouvêa and Y. Grossman, A three-flavor, Lorentzviolating solution to the LSND anomaly, Phys. Rev. D 74, 093008 (2006).

[27] T. Schwetz, LSND versus MiniBooNE: Sterile neutrinos with energy dependent masses and mixing? J. High Energy Phys. 02 (2008) 011.

[28] Y. Farzan, T. Schwetz, and A. Yu Smirnov, Reconciling results of LSND, MiniBooNE and other experiments with soft decoherence, J. High Energy Phys. 07 (2008) 067.

[29] S. Hollenberg, O. Micu, H. Pas, and T. J. Weiler, Baselinedependent neutrino oscillations with extra-dimensional shortcuts, Phys. Rev. D 80, 093005 (2009).

[30] A. E. Nelson, Effects of $C P$ violation from neutral heavy fermions on neutrino oscillations, and the LSND/MiniBooNE anomalies, Phys. Rev. D 84, 053001 (2011).

[31] E. Akhmedov and T. Schwetz, MiniBooNE and LSND data: Non-standard neutrino interactions in a $(3+1)$ scheme versus $(3+2)$ oscillations, J. High Energy Phys. 10 (2010) 115.

[32] J. S. Diaz and V. A. Kostelecky, Three-parameter Lorentzviolating texture for neutrino mixing, Phys. Lett. B 700, 25 (2011).

[33] Y. Bai, R. Lu, S. Lu, J. Salvado, and B. A. Stefanek, Three twin neutrinos: Evidence from LSND and MiniBooNE, Phys. Rev. D 93, 073004 (2016).

[34] C. Giunti and E. M. Zavanin, Appearance-disappearance relation in $3+N_{s}$ short-baseline neutrino oscillations, Mod. Phys. Lett. A 31, 1650003 (2016).

[35] D. K. Papoulias and T. S. Kosmas, Impact of nonstandard interactions on neutrino-nucleon scattering, Adv. High Energy Phys. 2016, 1490860 (2016).

[36] Z. Moss, M. H. Moulai, C. A. Argüelles, and J. M. Conrad, Exploring a nonminimal sterile neutrino model involving decay at IceCube, Phys. Rev. D 97, 055017 (2018).

[37] M. Carena, Y.-Y. Li, C. S. Machado, P. A. N. Machado, and C.E. M. Wagner, Neutrinos in large extra dimensions and short-baseline $\nu_{e}$ appearance, Phys. Rev. D 96, 095014 (2017).

[38] J. Liao and D. Marfatia, Impact of Nonstandard Interactions on Sterile Neutrino Searches at IceCube, Phys. Rev. Lett. 117, 071802 (2016).

[39] J. Liao, D. Marfatia, and K. Whisnant, MiniBooNE, MINOS+ and IceCube data imply a Baroque neutrino sector, Phys. Rev. D 99, 015016 (2019).

[40] J. Asaadi, E. Church, R. Guenette, B. J. P. Jones, and A. M. Szelc, New light Higgs boson and short-baseline neutrino anomalies, Phys. Rev. D 97, 075021 (2018).

[41] D. Döring, H. Päs, P. Sicking, and T. J. Weiler, Sterile neutrinos with altered dispersion relations as an explanation for the MiniBooNE, LSND, gallium and reactor anomalies, arXiv:1808.07460. 
[42] S. N. Gninenko, The MiniBooNE Anomaly and Heavy Neutrino Decay, Phys. Rev. Lett. 103, 241802 (2009).

[43] S. N. Gninenko, A resolution of puzzles from the LSND, KARMEN, and MiniBooNE experiments, Phys. Rev. D 83, 015015 (2011).

[44] C. Dib, J. C. Helo, S. Kovalenko, and I. Schmidt, Sterile neutrino decay explanation of LSND and MiniBooNE anomalies, Phys. Rev. D 84, 071301(R) (2011).

[45] D. McKeen and M. Pospelov, Muon capture constraints on sterile neutrino properties, Phys. Rev. D 82, 113018 (2010).

[46] M. Masip, P. Masjuan, and D. Meloni, Heavy neutrino decays at MiniBooNE, J. High Energy Phys. 01 (2013) 106.

[47] M. Masip and P. Masjuan, Heavy-neutrino decays at neutrino telescopes, Phys. Rev. D 83, 091301(R) (2011).

[48] S. N. Gninenko, New limits on radiative sterile neutrino decays from a search for single photons in neutrino interactions, Phys. Lett. B 710, 86 (2012).

[49] G. Magill, R. Plestid, M. Pospelov, and Yu-D. Tsai, Dipole portal to heavy neutral leptons, Phys. Rev. D 98, 115015 (2018).

[50] E. Bertuzzo, S. Jana, P. A. N. Machado, and R. Z. Funchal, Neutrino masses and mixings dynamically generated by a light dark sector, Phys. Lett. B 791, 210 (2019).

[51] E. Bertuzzo, S. Jana, P. A. N. Machado, and R. Z. Funchal, A dark neutrino portal to explain MiniBooNE, Phys. Rev. Lett. 121, 241801 (2018).

[52] P. Ballett, S. Pascoli, and M. Ross-Lonergan, $U(1)^{\prime}$ mediated decays of heavy sterile neutrinos in MiniBooNE, Phys. Rev. D 99, 071701 (2019).

[53] P. Ballett, M. Hostert, and S. Pascoli, Neutrino masses from a dark neutrino sector below the electroweak scale, Phys. Rev. D 99, 091701(R) (2019).

[54] P. Ballett, M. Hostert, and S. Pascoli, Dark neutrinos and a three portal connection to the Standard Model, arXiv: 1903.07589.

[55] L. B. Auerbach et al. (LSND Collaboration), Measurement of electron-neutrino-electron elastic scattering, Phys. Rev. D 63, 112001 (2001).

[56] M. Deniz et al. (TEXONO Collaboration), Measurement of $\bar{\nu}_{e}$-electron scattering cross-section with a $\mathrm{CsI}(\mathrm{Tl})$ scintillating crystal array at the Kuo-Sheng nuclear power reactor, Phys. Rev. D 81, 072001 (2010).

[57] G. Bellini et al., Precision Measurement of the ${ }^{7}$ Be Solar Neutrino Interaction Rate in Borexino, Phys. Rev. Lett. 107, 141302 (2011).

[58] J. Park, Ph. D. thesis, U. Rochester, 2013.

[59] E. Valencia et al. (MINERvA Collaboration), Constraint of the MINERvA medium energy neutrino flux using neutrino-electron elastic scattering, Phys. Rev. D 100, 092001 (2019).

[60] J. Park et al. (MINERvA Collaboration), Measurement of neutrino flux from neutrino-electron elastic scattering, Phys. Rev. D 93, 112007 (2016).

[61] E. Valencia-Rodriguez, Ph. D. thesis, Guanajuato U., 2016.

[62] K. De Winter et al. (CHARM-II Collaboration), A detector for the study of neutrino-electron scattering, Nucl. Instrum. Methods Phys. Res., Sect. A 278, 670 (1989).

[63] D. Geiregat et al. (CHARM-II Collaboration), Calibration and performance of the CHARM-II detector, Nucl. Instrum. Methods Phys. Res., Sect. A 325, 92 (1993).
[64] P. Vilain et al. (CHARM-II Collaboration), Precision measurement of electroweak parameters from the scattering of muon-neutrinos on electrons, Phys. Lett. B 335, 246 (1994).

[65] M. Pospelov and Yu-D. Tsai, Light scalars and dark photons in Borexino and LSND experiments, Phys. Lett. B 785, 288 (2018).

[66] M. Lindner, F. S. Queiroz, W. Rodejohann, and X.-J. Xu, Neutrino-electron scattering: General constraints on $Z^{\prime}$ and dark photon models, J. High Energy Phys. 05 (2018) 098.

[67] G. Magill, R. Plestid, M. Pospelov, and Yu-D. Tsai, Millicharged particles in neutrino experiments, Phys. Rev. Lett. 122, 071801 (2019).

[68] K. Abe et al. (T2K), Search for neutral-current induced single photon production at the ND280 near detector in T2K, J. Phys. G 46, 08LT01 (2019).

[69] J. A. Formaggio, J. M. Conrad, M. Shaevitz, A. Vaitaitis, and R. Drucker, Helicity effects in neutral heavy lepton decays, Phys. Rev. D 57, 7037 (1998).

[70] A. B. Balantekin, A. de Gouvêa, and B. Kayser, Addressing the Majorana vs. Dirac question with neutrino decays, Phys. Lett. B 789, 488 (2019).

[71] E. J. Chun, J.-C. Park, and S. Scopel, Dark matter and a new gauge boson through kinetic mixing, J. High Energy Phys. 02 (2011) 100.

[72] S. Parke and M. Ross-Lonergan, Unitarity and the three flavour neutrino mixing matrix, Phys. Rev. D 93, 113009 (2016).

[73] M. Bauer, P. Foldenauer, and J. Jaeckel, Hunting All the Hidden Photons, J. High Energy Phys. 07 (2018) 094.

[74] J. R. Jordan, Y. Kahn, G. Krnjaic, M. Moschella, and J. Spitz, Severe constraints on new physics explanations of the MiniBooNE excess, Phys. Rev. Lett. 122, 081801 (2019).

[75] See Supplemental Material at http://link.aps.org/ supplemental/10.1103/PhysRevLett.123.261801 for [brief description].

[76] M. Tanabashi et al. (Particle Data Group), Review of particle physics, Phys. Rev. D 98, 030001 (2018).

[77] L. Aliaga et al. (MINERvA Collaboration), Neutrino flux predictions for the NuMI beam, Phys. Rev. D 94, 092005 (2016); Publisher's Note: Neutrino flux predictions for the NuMI beam [Phys. Rev. D 94, 092005 (2016)], Phys. Rev. D 95, 039903 (2017).

[78] J. V. Allaby et al. (CHARM Collaboration), Total crosssections of charged current neutrino and anti-neutrino interactions on isoscalar nuclei, Z. Phys. C 38, 403 (1988).

[79] A. de Gouvea and J. Jenkins, What can we learn from neutrino electron scattering?, Phys. Rev. D 74, 033004 (2006).

[80] P. Adamson et al. (MINOS Collaboration), Measurement of single $\pi^{0}$ production by coherent neutral-current $\nu \mathrm{Fe}$ interactions in the MINOS near detector, Phys. Rev. D 94, 072006 (2016).

[81] J. Wolcott et al. (MINERvA Collaboration), Evidence for Neutral-Current Diffractive $\pi^{0}$ Production from Hydrogen in Neutrino Interactions on Hydrocarbon, Phys. Rev. Lett. 117, 111801 (2016). 